Published in final edited form as:

Conf Proc IEEE Eng Med Biol Soc. 2009 ; 2009: 1926-1929. doi:10.1109/IEMBS.2009.5333926.

\title{
Multimodal Functional Imaging Using fMRI-Informed Regional EEG/MEG Source Estimation
}

\author{
Wanmei Ou, \\ Computer Science and Artificial Intelligence Laboratory, MIT, USA
}

Aapo Nummenmaa,

Athinoula A. Martinos Center for Biomedical Imaging, MGH, USA

Polina Golland, and

Faculty of Computer Science and Artificial Intelligence Laboratory, MIT, USA

\section{Matti S. Hämäläinen}

Faculty of Athinoula A. Martinos Center for Biomedical Imaging, MGH, USA

Wanmei Ou: wanmei@csail.mit.edu; Aapo Nummenmaa: nummenma@nmr.mgh.harvard.edu; Polina Golland: polina@csail.mit.edu; Matti S. Hämäläinen:msh@nmr.mgh.harvard.edu

\section{Abstract}

We propose a novel method, fMRI-Informed Regional Estimation (FIRE), which utilizes information from fMRI in E/MEG source reconstruction. FIRE takes advantage of the spatial alignment between the neural and the vascular activities, while allowing for substantial differences in their dynamics. Furthermore, with the regional approach, FIRE can be efficiently applied to a dense grid of sources. Inspection of our optimization procedure reveals that FIRE is related to the re-weighted minimum-norm algorithms, the difference being that the weights in the proposed approach are computed from both the current estimates and fMRI data. Analysis of both simulated and human fMRI-MEG data shows that FIRE reduces the ambiguities in source localization present in the minimum-norm estimates. Comparisons with several joint fMRI-E/MEG algorithms demonstrate robustness of FIRE in the presence of sources silent to either fMRI or E/MEG measurements.

\section{Introduction}

The principal difficulty in interpreting Electroencephalography (EEG) and magnetoencephalography (MEG) data stems from the ill-posed electromagnetic inverse problem: infinitely many spatial current patterns give rise to identical measurements[8]. Therefore, additional assumptions must be incorporated into the reconstruction process to obtain a unique estimate [2].

In addition to the general assumptions about the spatial current patterns such as minimum energy (or $\ell_{2}$-norm), specific prior knowledge about activation locations can be obtained from other imaging modalities. Among them, functional Magnetic Resonance Imaging (fMRI) provides the most relevant information for the reconstruction due to its good spatial resolution. fMRI measures the hemodynamic activity, which indirectly reflects the neural activity measured by E/MEG. Extensive studies of neurovascular coupling have 
demonstrated similarity in spatial patterns of these two types of activations [11]. However, the timecourses of the neural and the vascular activities differ substantially, and their exact relationship has yet to be characterized in full [12]. In addition to the differences in their physiological origins, E/MEG and fMRI have different sensitivity characteristics. For example, a brief transient neural activity may be difficult to detect in fMRI while sustained weak neural activity may lead to relatively strong fMRI signals but might have a poor signal-to-noise ratio in E/MEG.

The most straightforward way to incorporate fMRI information into E/MEG inverse estimation is the fMRI-weighted Minimum-Norm Estimation (fMNE) [1], [10]. This method uses a thresholded Statistical Parametric Map (SPM) from fMRI analysis to construct weights for the standard Minimum-Norm Estimation (MNE), leading to significant improvements when the SPM is accurate. However, the weights depend on arbitrary choices of the threshold and weighting parameters. Moreover, these weights are assumed to be time independent causing excessive bias in the estimated source timecourses. Sato et al. [15] combined the Automatic Relevance Determination (ARD) framework and fMNE to achieve more focal estimates. In this method, which we will refer to as fARD, the parameters of a hyper-prior are set based on the thresholded SPM. In addition to the arbitrary choice of the threshold similar to that in fMNE, the estimates computed via fARD are often unstable, especially in the regions where the vascular activity is weak.

Here, we propose a novel method, the fMRI-Informed Regional Estimation (FIRE), to improve on the accuracy of the E/MEG source estimates. Since the relationship between the dynamics of the evoked neural and vascular signals is largely unknown, we only model the similarity of spatial patterns of the two processes, as opposed to the Kalman-filter approach in [5]. Furthermore, we expect that the shape of the activation timecourses varies across brain regions, especially for the neural activation timecourses. To account for this fact, FIRE treats the temporal dynamics in different brain regions independently. We assume the shape of the activation timecourses to be constant within a brain region, modulated by a set of location-specific latent variables. The regions are chosen based on a subject-specific cortical parcellation[6]. Handling the temporal dynamics of the two types of activities separately while exploring their common spatial pattern helps to preserve the temporal resolution of E/MEG and to achieve accurate source localization.

Both our activation timecourse model and the choice of brain regions in FIRE are similar to those employed in recent work by Daunizeau et al. [3]. However, Daunizeau et al. aim to symmetrically infer brain activities visible in either EEG or fMRI data, resulting in an extra random variable to model the vascular activity. Furthermore, due to the complexity of this model, the estimation is limited to source space that substantially coarser than the spatial resolution of fMRI. Instead of aiming at a symmetrical inference, we focus on the estimation of current sources. We incorporate the fMRI information to reduce ambiguities in source localization usually present in E/MEG source estimation.

To fit the model to the data, we employ the coordinate descent method, alternating between the estimation of current sources and of other model parameters. This iterative update scheme is similar to the re-weighted MNE methods such as FOCal Underdetermined System 
Solver (FOCUSS) [7]. In contrast to the re-weighted MNE, in our method the weights are jointly determined using both the estimated neural activity and the vascular activity measured by fMRI. Moreover, the estimates at different time points influence each other.

In the following, we first discuss the model underlying FIRE and the inference procedure. We then present the experimental comparisons between FIRE and previous models in joint E/MEG-fMRI analysis, followed by conclusions.

\section{Methods}

\section{A. Neurovascular coupling and data models}

We assume that the source space comprises $N$ discrete locations on the cortex parcelled into $K$ brain regions. We denote the set indexing the discrete locations in region $k$ by $P_{k}$ and the cardinality of $P_{k}$ by $N_{k}$.

Fig. 1 illustrates the structure of our model. The shape of the source timecourses is identical within a region but varies across regions. Specifically, we let $\mathbf{u}_{k}$ and $\mathbf{v}_{k}$ be the unknown waveforms in region $k$, associated with neural and vascular activities, respectively. We model the neural and the vascular activity strength through a hidden vector variable $\mathbf{Z}=$ $\left[\mathbf{z}_{1}, \mathbf{z}_{2}, \ldots, \mathbf{z}_{N}\right]^{\mathrm{T}}$. The continuous scalar $\mathbf{z}_{n}$ indicates the activation strength at location $n$ on the cortical surface. Thus, the probabilistic model for the neural activation timecourse $\mathbf{j}_{n}$ and the vascular activation timecourse $\mathbf{f}_{n}$ at location $n$ in region $k$ can be expressed as

$$
\begin{aligned}
& \log p\left(\mathbf{j}_{n}, \mathbf{f}_{n} \mid \mathbf{z}_{n} ; \mathbf{u}_{k}, \mathbf{v}_{k}, \eta_{k}^{2}, \xi_{k}^{2}\right) \\
= & \log \mathscr{N}\left(\mathbf{j}_{n} ; \mathbf{z}_{n} \mathbf{u}_{k}, \eta_{k}^{2} \mathbf{I}\right)+\log \mathscr{N}\left(\mathbf{f}_{n} ; \mathbf{z}_{n} \mathbf{v}_{k}, \xi_{k}^{2} \mathbf{I}\right)
\end{aligned}
$$

where $\eta_{k}^{2}$ and $\xi_{k}^{2}$ are noise variances. We construct all matrices such that each row represents a location or a sensor and each column represents a particular time point. Thus, we let $N \times$ $T_{\mathbf{J}}$ matrix $\mathbf{J}=\left[\mathbf{j}_{1}, \mathbf{j}_{2}, \ldots, \mathbf{j}_{N}\right]^{\mathrm{T}}$ be the neural current on the cortex for all $T_{\mathbf{J}}$ time points. We assume that the vascular signal $\mathbf{f}_{n}$ at location $n$ is directly observable through fMRI. We let $N \times T_{\mathbf{F}}$ matrix $\mathbf{F}=\left[\mathbf{f}_{1}, \mathbf{f}_{2}, \ldots, \mathbf{f}_{N}\right]^{\mathrm{T}}$ be the fMRI measurements on the cortex over $T_{\mathbf{F}}$ time points. Note that our neurovascular coupling model captures only the spatial alignment between the two types of activities; it does not impose temporal similarity between the signals.

The neural currents $\mathbf{j}_{n}$ are detected with E/MEG described by the standard observation model. We let $M \times T_{\mathbf{J}}$ matrix $\mathbf{Y}=\left[\mathbf{y}(1), \mathbf{y}(2), \ldots, \mathrm{y}\left(T_{\mathbf{J}}\right)\right]$ be the E/MEG measurements at all $T_{\mathbf{J}}$ time points. Column $t$ of matrix $\mathbf{J}, \mathbf{j}(t)$, denotes the neural currents at time $t$. The quasistatic Maxwell's equations imply that E/MEG signals at time $t$ are instantaneous linear combinations of the currents at different locations:

$$
\mathbf{y}(t)=\mathbf{A j}(\mathbf{t})+\mathbf{e}(\mathbf{t}) \quad \forall \mathbf{t}=\mathbf{1}, \mathbf{2}, \cdots, \mathbf{T}_{\mathbf{J}},
$$

where $\mathbf{e}(t)$ is the measurement noise. The $M \times N$ forward matrix $\mathbf{A}$ is determined by the electromagnetic properties of the head, the geometry of the sensors, and the set of potential 
locations of the sources. With spatial whitening in the sensor space, $\mathbf{e}(t) \sim \mathcal{N}(\mathbf{0}, \mathbf{I})$. The number of sources $N\left(\sim 10^{3}-10^{4}\right)$ is much larger than the number of measurements $M(\sim$ $\left.10^{2}\right)$, leading to an infinite number of solutions satisfying Eq. (2) even for $\mathbf{e}(t)=\mathbf{0}$. In general, $\mathbf{j}_{n}$ should be modeled as three timecourses corresponding to the three Cartesian components of the current. However, due to the columnar organization of the cortex, we can further constrain the current orientation to be perpendicular to the cortical surface and consider a scalar timecourse at each location.

\section{B. Priors and Parameter Settings}

To encourage the activation patterns to be smooth within a region, we impose a prior on the modulating variables. Specifically, we define $\underline{\mathbf{z}}_{k}=\left\{\mathbf{z}_{n}\right\}_{n \in P_{k}}$ and assume $\underline{\mathbf{z}}_{k} \sim \mathscr{N}\left(\mathbf{0}, \gamma_{k}^{2} \mathbf{\Phi}_{k}\right)$, where the variance $\gamma_{k}^{2}$ indicates the activation strength in region $k$, and $\Phi_{k}$ is a fixed matrix that acts as a regularizer by penalizing the sum of squared differences between neighboring locations. This spatial prior is particularly important for the brain regions where vascular activity is too weak to measure, but the neural activity can be detected by E/MEG.

Our $\Phi_{k}$ is similar to the regularizer used in the Low Resolution Brain Electromagnetic Tomography (LORETA) [14], except that we apply $\Phi_{k}$ to individual brain regions while

LORETA's spatial regularizer is applied to the whole brain. We assume separate variance $\gamma_{k}^{2}$ for different brain regions since the strength of current is expected to vary significantly between regions with and without active sources. This choice is similar to the recent work in the application of ARD to E/MEG reconstruction [15], [16], except that their work assumes independent $\gamma^{2}$ for each location in the brain.

Since the forward model $\mathbf{A}$ is underdetermined, the current distribution $\mathbf{J}$, produced by our neurovascular coupling model, can fully explain the E/MEG data. In other words, without the noise term $\eta_{k}^{2}$ (i.e., $\mathbf{j}_{n}=\mathbf{z}_{n} \mathbf{u}_{k}$ ), the fMRI data can exert too much influence on the reconstruction results. Although we can estimate the noise variance of the current source timecourses $\eta_{k}^{2}$ by extending the inference procedure, we find the corresponding estimate unstable without a prior. Based on preliminary empirical testing, we fix $\eta_{k}^{2}=1$. With proper temporal whitening of the fMRI data, we can also assume that $\xi_{k}^{2}=\eta_{k}^{2}$. Fixing $\eta_{k}^{2}=\xi_{k}^{2}$ helps to significantly reduce the computational burden of the estimation.

To summarize, our model can be expressed as

$$
p(\mathbf{F}, \boldsymbol{Y}, \mathbf{J}, \mathbf{z} ; \boldsymbol{\Theta})=p(\boldsymbol{Y} \mid \mathbf{J}) p(\mathbf{F}, \mathbf{J} \mid \boldsymbol{Z} ; \boldsymbol{\Theta}) p(\boldsymbol{Z} ; \boldsymbol{\Theta})
$$

where $\Theta=\left[\theta_{1}, \theta_{2}, \cdots, \theta_{K}\right]$ is the combined set of parameters, and $\theta_{k}=\left\{\mathbf{u}_{k}, \mathbf{v}_{k}, \gamma_{k}^{2}\right\} . p(\mathbf{Y} \mid \mathbf{J})$ is the E/MEG data model in Eq. (2). $p(\mathbf{F}, \mathbf{J} \mid \mathbf{Z} ; \Theta)$ is our neurovascular coupling model in Eq. $(1)$, and $p(\mathbf{Z} ; \boldsymbol{\Theta})$ is the prior on $\mathbf{Z}$. 


\section{Inference}

Our goal is to estimate the current source $\mathbf{J}$ and the timecourses $\mathbf{u}$ and $\mathbf{v}$. The activation strength $\mathbf{Z}$ is considered as an auxiliary variable, and is marginalized in the analysis. We formulate the inference as

$$
\begin{aligned}
\{\mathbf{J} *, \boldsymbol{\Theta} *\} & =\underset{\mathbf{J}, \boldsymbol{\Theta}}{\arg \max } \log p(\mathbf{F}, \boldsymbol{Y}, \mathbf{J} ; \boldsymbol{\Theta}) \\
& =\underset{\mathbf{J}, \boldsymbol{\Theta}}{\arg \max } \log \int_{\boldsymbol{Z}} p(\mathbf{F}, \boldsymbol{Y}, \mathbf{J}, \boldsymbol{Z} ; \boldsymbol{\Theta}) d \boldsymbol{Z} \\
& =\underset{\mathbf{J}, \boldsymbol{\Theta}}{\arg \max l o g}(p(\boldsymbol{Y} \mid \boldsymbol{Z}) p(\mathbf{F}, \mathbf{J} ; \boldsymbol{\Theta})) .
\end{aligned}
$$

With marginalization of $\mathbf{Z}, p(\mathbf{F}, \mathbf{J} ; \boldsymbol{\Theta})$ acts as the prior for $\mathbf{J}$. Since both $\mathbf{F}$ and $\mathbf{J}$ are linear functions of $\mathbf{Z}, p(\mathbf{F}, \mathbf{J} ; \Theta)$ is a continuous Gaussian mixture model.

The difficulty in the inference of the proposed model is caused by the intertwining between space and time, reflected by the intersection of the temporal plates and the spatial plates in Fig. 1. That is because the output of a given E/MEG sensor is a mixture of signals from the entire source space. Moreover, with $\mathbf{Z}$ marginalized, $\mathbf{F}, \mathbf{J}$, and $\mathbf{Y}$ are jointly Gaussian distributed. The correlation between different time points (i.e., between two E/MEG time points, between two fMRI time points, and between E/MEG and fMRI time points) is generally not zero. Hence, the inference must be performed for all time points and all locations simultaneously. FIRE is thus substantially more computationally demanding compared to standard point-wise E/MEG estimation in the time domain and voxel-wise fMRI analysis.

Due to the special structure in our model, we can derive an efficient gradient descent method with two alternating steps. In the first step, we fix $\Theta$ and derive a closed-form solution for J. In the second step, we fix $\mathbf{J}$ and show that $\Theta$ can be efficiently estimated through the Expectation-Maximization (EM) algorithm [4].

For a fixed $\Theta=\hat{\Theta}, p(\mathbf{F}, \mathbf{Y}, \mathbf{J} ; \hat{\Theta})$ is a jointly-Gaussian distribution. Thus, the estimate of $\mathbf{J}$ is the conditional mean:

$$
\begin{aligned}
\hat{\mathbf{J}} & =\underset{\mathbf{J}}{\arg \max } \log p(\mathbf{F}, \boldsymbol{Y}, \mathbf{J} ; \hat{\boldsymbol{\Theta}}) \\
& =E[\mathbf{J} \mid \boldsymbol{Y}, \mathbf{F} ; \hat{\boldsymbol{\Theta}}]=\boldsymbol{\Gamma}_{\mathbf{W}, \mathbf{J}}^{\mathbf{T}} \boldsymbol{\Gamma}_{\mathbf{W}}^{-1} \mathbf{W},
\end{aligned}
$$

where $\mathbf{W}^{\mathrm{T}}=\left[(\operatorname{vec}(\mathbf{Y}))^{\mathrm{T}}(\operatorname{vec}(\mathbf{F}))^{\mathrm{T}}\right]$ includes both E/MEG and fMRI measurements. Operator vec $(\cdot)$ concatenates adjacent columns of a matrix. $\Gamma_{\mathrm{W}}$ is the covariance matrix of $\mathbf{W}$, and $\Gamma_{\mathbf{W}, \mathbf{J}}$ is the cross-covariance matrix between $\mathbf{W}$ and vec $(\mathbf{J})$. Thus, E/MEG and fMRI measurements jointly determine the estimate of the neural activity. Eq. (5) is similar to the standard MNE solution [8], but it also includes the correlation between $\mathbf{Y}$ and $\mathbf{F}$ and the correlation among different time points of $\mathbf{J}$.

For a fixed $\mathbf{J}=\hat{\mathfrak{J}}$, we optimize the parameters $\Theta$ : 


$$
\hat{\mathbf{\Theta}}=\underset{\boldsymbol{\Theta}}{\arg \max } \log p(\mathbf{F}, \hat{\mathbf{J}} ; \boldsymbol{\Theta}) .
$$

As shown in Fig. 1, when the current distribution $\mathbf{J}$ is fixed, the E/MEG measurement $\mathbf{Y}$ does not provide additional information for the parameter estimation. Since the parameters for different regions are independent for a fixed $\hat{\jmath}$, the estimates for different regions can be obtained independently. Furthermore, parameter $\Theta$ can be efficiently estimated using the EM algorithm by re-introducing the latent variable $\mathbf{Z}$, which is the auxiliary variable describing the activation strength. For region $k$, the parameter estimates $\hat{\theta}_{k}$ can be obtained by optimizing the lower bound of the log-probability:

$$
\begin{gathered}
\log p\left(\left\{\mathbf{f}_{n}, \hat{\mathbf{j}}_{n}\right\}_{n \in P_{k}} ; \theta_{k}\right) \\
\geq \int_{\underline{\mathbf{z}}_{k}} q\left(\underline{\mathbf{z}}_{k}\right) \log p\left(\left\{\mathbf{f}_{n}, \hat{\mathbf{j}}_{n}\right\}_{n \in P_{k}}, \underline{\mathbf{z}}_{k} ; \theta_{k}\right) d \underline{\mathbf{z}}_{k},
\end{gathered}
$$

where $q\left(\underline{\mathbf{z}}_{k}\right)=p\left(\underline{\mathbf{z}}_{k} \mid\left\{\mathbf{f}_{n}, \hat{\mathbf{j}}_{n}\right\}_{n \in P_{k}} ; \hat{\theta}_{k}\right)$ is the posterior probability computed in the E-step. Since $\left\{\mathbf{f}_{n}, \hat{\mathbf{j}}_{n}\right\}_{n \in P_{k}}$ and $\underline{\mathbf{z}}_{k}$ are jointly-Gaussian distributed for a fixed $\hat{\theta}_{k}, q\left(\underline{\mathbf{z}}_{k}\right)$ is also a Gaussian distribution. We use $\langle\cdot\rangle_{q}$ to denote the expectation with respect to the posterior distribution $q\left(\underline{\mathbf{z}}_{k}\right)$, i.e., $\langle\cdot\rangle_{q}=E\left[\cdot \mid\left\{\mathbf{f}_{n}, \hat{\mathbf{j}}_{n}\right\}_{n \in P_{k}} ; \hat{\theta}_{k}\right]$. Since the M-step depends only on quantities related to the first- and the second-order statistics of $\underline{\mathbf{z}}_{k}$, we only need to update quantities $\left\langle\underline{\mathbf{z}}_{k} \underline{\mathbf{z}}_{k}^{T}\right\rangle q,\left\langle\underline{\mathbf{z}}_{k}\right\rangle_{q}$, and $\left\langle\underline{\mathbf{z}}_{k}^{\mathrm{T}} \boldsymbol{\Phi}_{k}^{-1} \underline{\mathbf{z}}_{k}\right\rangle q$ in the E-step. The detailed derivations can be found in [13];

In the M-step, we fix $q\left(\underline{\mathbf{z}}_{k}\right)$ and optimize Eq.(7). With some algebra, we arrive at the update equations for the model parameters:

$$
\begin{gathered}
\hat{\mathbf{u}}_{k} \leftarrow \frac{\sum_{n \in P_{k}}\left\langle\mathbf{z}_{n}\right\rangle_{q} \hat{\mathbf{j}}_{n}}{\operatorname{tr}\left(\left\langle\underline{\mathbf{z}}_{k} \underline{\mathbf{z}}_{k}^{T}\right\rangle_{q}\right)}, \hat{\mathbf{v}}_{k} \leftarrow \frac{\sum_{n \in P_{k}}\left\langle\mathbf{z}_{n}\right\rangle_{q} \mathbf{f}_{n}}{\operatorname{tr}\left(\left\langle\underline{\mathbf{z}}_{k} \mathbf{\mathbf { z }}_{k}^{\mathrm{T}}\right\rangle_{q}\right)}, \\
\quad \text { and } \hat{\gamma_{k}^{2}} \leftarrow \frac{\left\langle\underline{\mathbf{z}}_{k}^{T} \boldsymbol{\Phi}_{k}^{-1} \underline{\mathbf{z}}_{k}\right\rangle_{q}}{N_{k}} .
\end{gathered}
$$

We iterate the EM algorithm until convergence which usually takes less than ten iterations. We then re-estimate $\mathbf{J}$ according to Eq. (5).

To summarize, the algorithm proceeds as follows:

i. Initialize \&Jcirc; as the MNE estimate.

ii. Until convergence:

1. Compute $\hat{\Theta}$ using the EM algorithm: E-step for the hidden variable $\mathbf{Z}$ followed by M-step for the model parameters $\Theta$.

2. Update $\hat{\jmath}$ according to Eq. (5) for $\Theta=\hat{\Theta}$. 


\section{Results}

We compared the performance of MNE, fMNE, fARD, and FIRE using simulated data and MEG-fMRI data from a somatosensory study. Due to space limitation, we only report the results of the somatosensory study here. In this experiment, the median nerve at the right wrist was stimulated according to an event-related protocol, with a random inter-stimulusinterval ranging from 3 to $14 \mathrm{~s}$. This stimulus activates a complex cortical network [9], including the contralateral primary somatosensory cortex (cSI) and bilateral secondary somatosensory cortices (cSII and iSII).

The MEG measurements were acquired using a 306-channel Neuromag VectorView MEG system. A 200-ms baseline before the stimulus was used to estimate the noise covariance matrix of the MEG sensors. An average signal, computed from approximately 100 trials, was used as the input to each method. In a separate session, the fMRI images were acquired using a Siemens $3 \mathrm{~T}$ machine $\left(\mathrm{TR}=1.5 \mathrm{~s}, 64 \times 64 \times 24,3 \times 3 \times 6 \mathrm{~mm}^{3}\right)$. Anatomical images, from a 3T scanner, were used to construct the cortical source space and the boundary-element forward model. We also obtained the cortical parcellation using the Freesurfer software, with 35 regions per hemisphere.

In the leftmost column in Fig. 2, approximate locations for cSI (solid), cSII (dashed), and iSII (dashed) are highlighted on the fMRI activation maps ( $p \leq 0.005)$. Note that in the noisy SPM, the sites of fMRI activations do not exactly agree with the locations of the expected current sources.

Columns two to five in Fig. 2 present the estimates from one subject at $75 \mathrm{~ms}$ after stimulus onset, during which cSI, cSII, and iSII should be activated. Since the activation in iSII is much weaker than that in cSI and cSII, the threshold was set separately for each hemisphere. For each method, the threshold is set to be 1/6 of the maximum absolute value of the corresponding current estimates. MNE produces a more diffuse estimate, including physiologically unlikely activations at the gyrus anterior to the cSI area. In contrast, FIRE pinpoints cSI on the post-central gyrus. With the prior knowledge from fMRI, the detected cSII and iSII activations using fMNE, fARD, and FIRE are within the expected areas. The fMNE and fARD show stronger weighting towards the fMRI, reflected by the activations in the temporal lobes. Due to the highly folded cortex and uncertainties in MRI-fMRI registration, fMRI cannot distinguish between the walls of the central sulcus and the postcentral sulcus, causing both walls to show strong vascular activity after mapping of the fMRI volume onto the cortex. Hence, fMNE, fARD, and FIRE estimates extend to both sulcal walls mentioned above.

\section{Conclusions}

In contrast to most joint fMRI-E/MEG models, FIRE explicitly takes into account the inherent differences in the data measured by E/MEG and fMRI. The corresponding estimates can be efficiently computed with an iterative procedure which bears similarity with re-weighted MNE methods, except that the weights are based on both the current estimates from the previous iteration and the fMRI data via the proposed neurovascular coupling model. This construction of the weights reduces the excessive sensitivity to fMRI 
present in many joint fMRI-E/MEG analysis methods, leading to more accurate current estimates as demonstrated by analysis of both simulated and human data.

\section{Acknowledgments}

We thank Dr. Raij and Dr. Siracusa for stimulating discussion. This work was supported in part by NIH NIBIB NAMIC U54-EB005149, NIH NCRR NAC P41-RR13218, NIH NCRR P41-RR14075 grants, and the NSF CAREER Award 0642971. Wanmei Ou is partially supported by the PHS training grant DA022759-03.

\section{References}

1. Ahlfors S, Simpson G. Geometrical interpretation of fMRI-guided MEG/EEG inverse estimates. NeuroImage. 2004; 22:323-32. [PubMed: 15110022]

2. Baillet S, et al. Electromagnetic brain mapping. IEEE Sig Proc Mag. 2001

3. Daunizeau J, et al. Symmetrical event-related EEG/fMRI information fusion in a variational Bayesian framework. NeuroImage. 2007; 36:69-87. [PubMed: 17408972]

4. Dempster A, et al. Maximum likelihood from incomplete data via the EM algorithm. J of Roy Stat Soc B. 1977; 39:1-38.

5. Deneux T, Faugeras O. EEG-fMRI fusion of non-triggered data using Kalman filtering. In Proc ISBI. 2006:1068-17.

6. Fischl B, et al. Whole brain segmentation: automated labeling of neuroanatomical structures in the human brain. Neuron. 2002; 33:341-55. [PubMed: 11832223]

7. Gorodnitsky I, Rao B. Sparse signal reconstruction from limited data using FOCUSS: a re-weighted minimum norm algorithm. IEEE Trans Sig Proc. 1997; 45:600-16.

8. Hämäläinen M, et al. Magnetoencephalography - theory, instrumentation, and applications to noninvasive studies of the working human brain. Rev Mod Phys. 1993; 65:413-97.

9. Hari R, Forss N. Magnetoencephalography in the study of human somatosensory cortical processing. Philos Trans R Soc Lond B. 1999; 354:1145-54. [PubMed: 10466142]

10. Liu A, et al. Spatiotemporal imaging of human brain activity using functional MRI constrained magnetoencephalography data: Monte Carlo simulations. PNAS. 1998; 95:8945-50. [PubMed: 9671784]

11. Logothetis N, Wandell B. Interpreting the BOLD signal. Annu Rev Physiol. 2004; 66:735-69. [PubMed: 14977420]

12. $\mathrm{Ou} \mathrm{W}$, et al. Study of neurovascular coupling in humans via simultaneous Magnetoencephalography and diffuse optical imaging acquisition. NeuroImage. 2009; 46:624-32. [PubMed: 19286463]

13. Ou W, et al. Multimodal functional imaging using fMRI-informed regional EEG/MEG source estimation. Proc IPMI, LNCS. 2009; 5636:88-100.

14. Pascual-Marqui R, et al. Low resolution electromagnetic tomography: a new method for localizing electrical activity in the brain. Int J Psychophysiol. 1994; 18:49-65. [PubMed: 7876038]

15. Sato M, et al. Hierarchical Bayesian estimation for MEG inverse problem. NeuroImage. 2004; 23:806-26. [PubMed: 15528082]

16. Wipf D, Nagarajan S. A unified Bayesian framework for MEG/EEG source imaging. NeuroImage. 2009; 44:947-66. [PubMed: 18602278] 


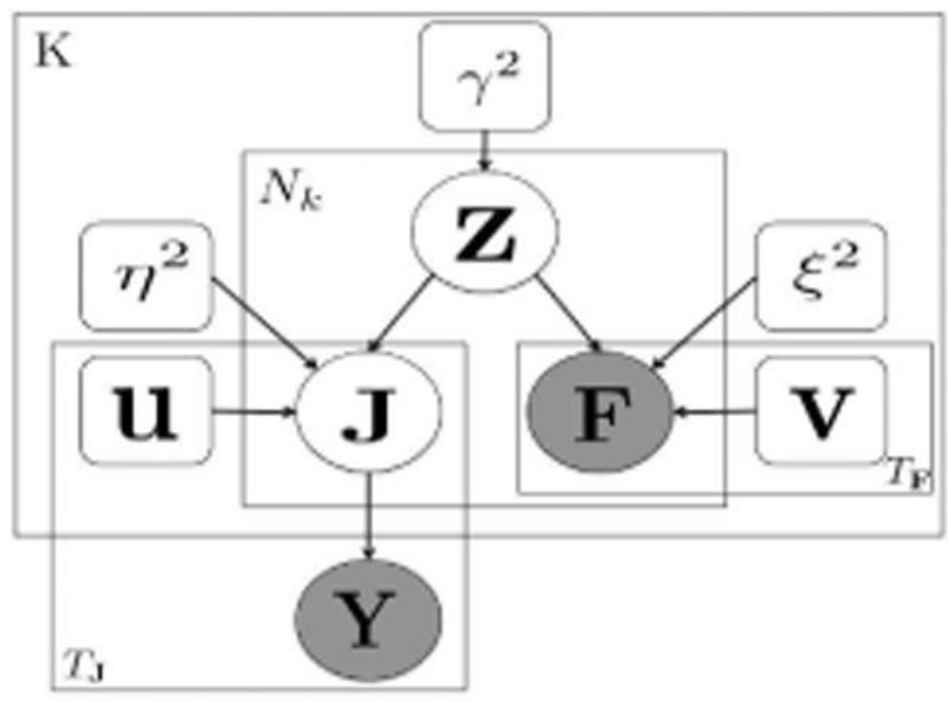

Fig. 1.

Graphical interpretation of FIRE. The hidden activity $\mathbf{z}$ models the neurovascular coupling relation. The hidden current source distribution $\mathbf{J}$ is measured by E/MEG, producing observation $\mathbf{Y}$. F denotes fMRI measurements. Vectors $\mathbf{u}$ and $\mathbf{v}$ are the unknown neural and vascular waveforms in a certain brain region, respectively. The inner plate represents $N_{k}$ vertices in region $k$; the outer plate represents $K$ regions. The bottom left and the bottom right plates represent $T_{\mathbf{J}}$ and $T_{\mathbf{F}}$ time points in the neural and the vascular measurements, respectively. 


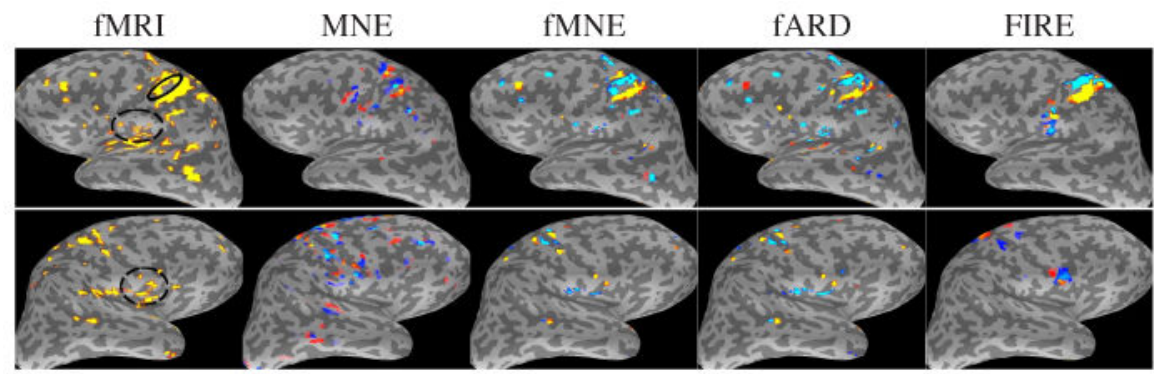

Fig. 2.

Human median-nerve experiments. In the first column, approximate locations for cSI (solid), cSII (dashed), and iSII (dashed) are highlighted on the fMRI activation maps. Columns two to five show the current estimates obtained via MNE, fMNE, fARD, and FIRE at $75 \mathrm{~ms}$ after stimulus onset. Hot/cold colors indicate outward/inward current flow. 